\title{
52. $\alpha_{-L}$-Fucosidase Polymorphism in Human Urine revealed by Isoelectric Focusing
}

\author{
By Koichiro KIsHI and Toshihiro YASUdA \\ Department of Legal Medicine, Fukui Medical School, \\ Shimoaizuki, Matsuoka-cho, Yoshida-gun, Fukui 910-11 \\ (Communicated by Shoei IsekI, M. J. A., April 12, 1985)
}

A polymorphism of $\alpha$-L-fucosidase has been found in human leucocytes. ${ }^{1), 2)}$ $\alpha$-L-Fucosidase has been demonstrated in serum, leucocytes, kidney, liver, lung, spleen, placenta and muscle, ${ }^{3}$ and in purified extracts of urine, serum and vaginal fluids. ${ }^{4), 5)}$

$\alpha$-L-Fucosidase is an important enzyme not only because its activity is deficient in the neurovisceral storage disease fucosidosis, ${ }^{6}$ ) but also because the enzyme is useful for structural studies on the blood group $A, B$, and $\mathrm{H}$ substances. $\left.{ }^{\top}\right), 8$ )

This paper presents new experimental evidence for $\alpha$-L-fucosidase polymorphism in human urine taken from unrelated individuals and from members of normal families.

Materials and methods. (1) Urine samples were collected from healthy Japanese individuals from 5-70 years of age. Urine was concentrated by Diaflo ultrafiltration cells (Amicon) with PM 10 membranes. ${ }^{9), 10)}$ The concentrated urine was dialyzed against $0.1 \%$ glycine overnight. The dialyzate was centrifuged and the supernatant was lyophilized. Obtained material was dissolved in distilled water at a concentration of $5 \%$ for electrophoresis. (2) $0.5 \times 90 \times 120 \mathrm{~mm}$ polyacrylamide gels were made as follows: $1.4 \mathrm{ml}$ acrylamide-bis $(20 \mathrm{~T}, 3 \mathrm{C}), 2.3$ $\mathrm{ml}$ sucrose-glycerin $(20 \% \mathrm{w} / \mathrm{v}, 10 \% \mathrm{v} / \mathrm{v}), 1 \mathrm{ml}$ distilled water, $240 \mu \mathrm{l}$ Ampholine 5-7 (LKB), $40 \mu \mathrm{l}$ Ampholine 3.5-10 (LKB), $5 \mu \mathrm{l}$ TEMED, and $40 \mu \mathrm{l} 1.2 \%$ ammonium persulfate. Wicks were formed from strips of filter paper and soaked in the electrode solution, $0.5 \mathrm{M} \mathrm{H}_{3} \mathrm{PO}_{4}$ at the anode and $0.5 \mathrm{M} \mathrm{NaOH}$ at the cathode. An LKB Multiphor apparatus was used to run the gel at $3 \mathrm{~W}$ for $4 \mathrm{hr}$. Ten $\mu$ l of each urine sample was applied to LKB sample applicater $2 \mathrm{~cm}$ from the anode on the gel. After electrophoresis the gel was incubated with $0.2 \mathrm{mg} / \mathrm{ml}$ 4-methyl-umbelliferyl- $\alpha$-L-fucopyranoside (Nakarai) in $0.1 \mathrm{~m}$ citric acid/phosphate buffer, $\mathrm{pH} 4.8$ for $15 \mathrm{~min}$ at $37^{\circ} \mathrm{C}$ using a cellulose acetate membrane overlay technique. Visualization occurred by fluorescence in a chamber containing ammonia gas under long-wave ultraviolet light. pI values of the enzymes were determined using a Pharmacia pI marker kit. (3) Neuraminidase treatment was performed as follows. A mixture containing $20 \mu \mathrm{l}$ of sample, $10 \mu \mathrm{l}$ of neuraminidase (Calbiochem, from Vibrio cholerae, $500 \mathrm{U} / \mathrm{ml}$ ) and $10 \mu \mathrm{l}$ of $10 \mathrm{~mm}$ citric acid/phosphate buffer, $\mathrm{pH} 4.8$ was incubated at room temperature overnight. 4) Leucocyte pellets prepared from heparinized blood samples of 4-5 ml have been prepared by gradient centrifugation on Ficoll-Paque (Pharmacia).

Results and discussion. 1) Electrophoresis of urinary $\alpha$-L-fucosidase. Isoelectric focusing in thin layer polyacryamide gel gave good separations of the different isozymes. Three different phenotypes $\alpha$ FUC 1, $\alpha$ FUC 2-1, and $\alpha$ FUC 2 were observed in our samples. The isozyme patterns could reliably be detected 
A

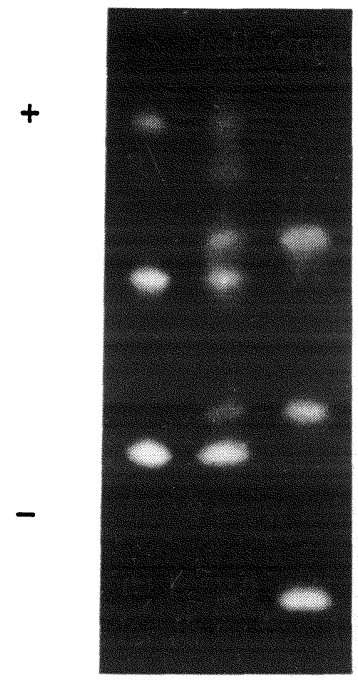

a $\quad b \quad c$
B
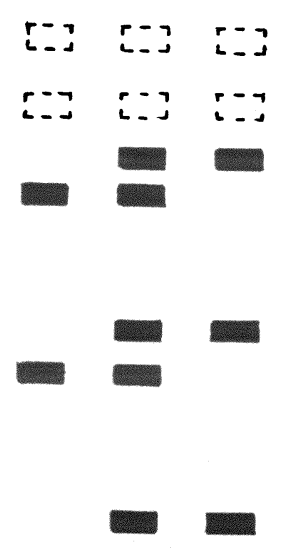

a $\quad b \quad c$

Fig. 1. A: Three different phenotypes $\alpha$ FUC 1, $\alpha$ FUC 2-1, and $\alpha$ FUC 2 of human urinary $\alpha$-L-fucosidase isozymes observed by isoelectric focusing. B: Schematic patterns. Lane a, $\alpha$ FUC 1; Lane b, $\alpha$ FUC 2-1; Lane c, $\alpha$ FUC 2 .

(Fig. 1). In this study we have shown that the isozyme profile of normal urinary $\alpha$-L-fucosidase is very constant and reproducible from childhood through adulthood. Homozygotes showed at least 4 bands of activity in urine. The leucocyte enzyme showed a similar picture. The $\alpha$ FUC 2-1 phenotype seemed to represent a simple mixture of $\alpha$ FUC 1 and $\alpha$ FUC 2. The phenotypes of $\alpha$-L-fucosidase in urine and leucocyte from the same individual of ten were virtually identical.

2) Family studies. The mode of inheritance of $\alpha$-L-fucosidase phenotypes was studied in 15 families with 31 children. In our samples the mating $\alpha$ FUC 2 $\times \alpha$ FUC 2 and $\alpha$ FUC $2-1 \times \alpha$ FUC 2 were not observed. The family data are given in Table I. This data show that polymorphism of the enzyme may be explained by the existence of two common alleles, $\alpha F U C^{1}$ and $\alpha F U C^{2}$, as reported previously. ${ }^{2)}$

Table I. Segregation of the urinary $\alpha$ FUC phenotypes

\begin{tabular}{ccccccc}
\hline \multirow{2}{*}{ Matings } & \multicolumn{2}{c}{ Number of } & & \multicolumn{3}{c}{ Children's phenotypes } \\
\cline { 2 - 3 } & families & children & 1 & $2-1$ & 2 \\
$1 \times 1$ & 5 & 10 & & 10 & 0 & 0 \\
$1 \times 2-1$ & 5 & 10 & & 4 & 6 & 0 \\
$1 \times 2$ & 3 & 7 & & 0 & 7 & 0 \\
$2-1 \times$ N.T. ${ }^{*}$ & 2 & 4 & & 1 & 1 & 2 \\
Total & 15 & 31 & & 15 & 14 & 2 \\
\hline
\end{tabular}

* Not tested because one parent was unavailable for this study. But one mating of the two was judged to be $2-1 \times 2-1$ from the children's phenotypes. 
Table II. Distribution of urinary $\alpha$ FUC phenotypes in a Japanese population

\begin{tabular}{cccccc}
\hline No. tested & \multicolumn{3}{c}{ Phenotypes } & \multicolumn{2}{c}{ Allele frequencies } \\
\cline { 5 - 6 } & 2 & $2-1$ & 2 & $\alpha F U C^{1}$ & $\alpha F U C^{2}$ \\
Observed 156 & 97 & 45 & 14 & 0.766 & 0.234 \\
Expected 156 & 91.5 & 55.9 & 8.6 & & \\
\hline$\left(\chi^{2}=5.8,1 \mathrm{df}, \mathrm{P}>0.01.\right)$ & & & &
\end{tabular}

3) Distribution of phenotypes. Urine samples of 156 Japanese were analyzed and the distribution of phenotypes observed in our samples is shown in Table II. The agreement between the observed and expected phenotypes is close. Frequencies of the $\alpha F U C^{1}$ and $\alpha F U C^{2}$ alleles were 0.766 and 0.234 , respectively. Frequencies of $\alpha F U C^{1}$ in white populations determined from leucocytes were $0.64-0.75,{ }^{2), 4), 11)}$ and in a Japanese population 0.7831.12)

4) Aging of samples. Some of lyophilized or dissolved urine samples were stored for about 5 years at $-20^{\circ} \mathrm{C}$ and tested. All of the aged samples showed enough enzyme activity and their phenotypes could be classified reliably. Activity of urinary $\alpha$-L-fucosidase in $5 \%$ sample solution, in correspondence to about 500-fold concentration of original urine, was stronger and more stable compared with that of the leucocyte enzyme. Therefore, the $\alpha$-L-fucosidase in urine was found to be a much more useful factor than the one in leucocytes in genetic studies.

5) Characteristics of urinary $\alpha$-L-fucosidase. The activity of $\alpha$-L-fucosidase in concentrated urine and semen was noticeably higher than that in leucocytes and serum. Neuraminidase treatment reduced the intensity of anodal bands in urine and caused pI shifts towards the cathode. This treatment caused a remarked reduction of the enzyme activities, and so was found not to aid identification of the three phenotypes of the urinary enzyme.

Summary. A polymorphism of $\alpha$-L-fucosidase has been found in human urine by isoelectric focusing technique. Three phenotypes $\alpha$ FUC 1, $\alpha$ FUC 2-1, and $\alpha$ FUC 2 were determined by two common alleles, $\alpha F U C^{1}$ and $F U C^{2}$. Paired leucocyte and urine samples from the same individuals were analyzed, and it was found that the phenotypes of the pairs were identical. Estimated frequencies of $\alpha F U C^{1}$ and $\alpha F U C^{2}$ among Japanese were 0.766 and 0.234 , respectively.

Acknowledgement. The excellent technical assistance of N. Sasahara is gratefully acknowledged.

\section{References}

1) Turner, B. M. et al.: Isozymes of human $\alpha$-L-fucosidase detectable by starch gel electrophoresis. Clin. Chim. Acta, 57, 29-35 (1974).

2) Turner, B. M. et al.: Polymorphism of human $\alpha$ fucosidase. Am. J. Hum. Genet., 27, 651-661 (1975).

3) Beyer, E. M., and Wiederschain, G. Y.: Further evidence of human $\alpha$-L-fucosidase polymorphism. Clin. Chim. Acta, 123, 251-259 (1982).

4) Gill, P., and Sutton, J. G.: $\alpha$-L-Fucosidase polymorphism in human semen, blood, and vaginal fluid. Hum. Hered., 34, 231-239 (1984).

5) Troost, J., Van der Heijden, M. C. M., and Staal, G. E. J.: Human leucocyte $\alpha$-L-fucosidase. Clin. Chim. Acta, 73, 321-327 (1976). 
6) Van Hoof, F., and Hers, H. G.: The abnormalities of lysosomal enzymes in mucopolysaccharidoses. Eur. J. Biochem., 7, 34-44 (1968).

7) Iseki, S., and Tsunoda, S.: On a bacterial enzyme which specifically decomposes O substance. Proc. Japan Acad., 28, 370-373 (1952).

8) Iseki, S.: Glycosidases and serological changes in blood group substances. Blood and Tissue Antigens (ed. Aminoff, D.). Academic Press, N.Y., pp. 379-394 (1970).

9) Kishi, K. et al.: Investigations of genetic variants in human urinary glycoproteins by isoelectric focusing. Proc. Japan Acad., 57B, 410-412 (1981).

10) Kishi, K., and Iseki, S.: Young age-related glycoproteins revealed in normal human urine by isoelectric focusing. ibid., 58B, 229-231 (1982).

11) Khoi, T.-D. et al.: Genetic polymorphism of $\alpha$-L-fucosidase in Brittany (France). Hum. Genet., 51, 293-296 (1979).

12) Toyomasu, T., Suzuki, K., and Matsumoto, H.: Genetic polymorphism of $\alpha$-fucosidase in a Japanese population. Act. Crim. Japon., 50, 11-12 (1984). 